Nachrichten der Deutschen Gesellschaft für Nuklearmedizin

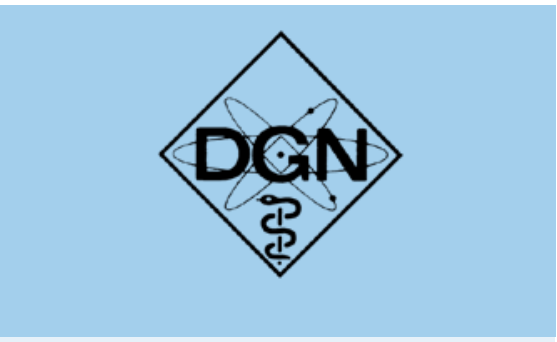

\title{
DGN und DGP unterschreiben Grundsatzvereinbarung
}

Um die Beziehungen zwischen den Fachgebieten zu vertiefen und einen regelmäßigen Gedanken- und Erfahrungsaustausch zu fördern, haben im Rahmen der Eröffnungsveranstaltung der Jahrestagung der DGN in Bremen die Deutsche Gesellschaft für Nuklearmedizin e. V. (DGN) und die Deutsche Gesellschaft für Pathologie e. V. (DGP) am 4. April 2019 eine Grundsatzvereinbarung über eine interdisziplinäre Zusammenarbeit in den Fachgebieten Nuklearmedizin und Pathologie unterschrieben.

Neben der Entwicklung von gemeinsamen Zukunftsstrategien möchten die beiden Fachgesellschaften hierdurch vor allem gemeinsame Standards auf dem Gebiet der molekularen Methoden und Analysen von Krankheits- und Therapiemechanismen definieren sowie die translationale Forschung für eine verbesserte Patientenversorgung fördern.
Dies soll unter anderem durch eine gegenseitige strategische Unterstützung als klinische Querschnittsfächer und eine Zusammenarbeit von DGN und DGP sowohl in der Leitlinienarbeit wie auch als Mitglied der Arbeitsgemeinschaft der Wissenschaftlichen Medizinischen Fachgesellschaften e. V. (AWMF) und der Deutschen Krebsgesellschaft (DKG) möglich werden. Darüber hinaus ist die Etablierung einer paritätisch besetzten Task Force zur Entwicklung gemeinsamer zukünftiger Strategien und zusätzlich eine inhaltlich enge Zusammenarbeit bei der Organisation von Jahrestagungen und weiterer Veranstaltungen der jeweils anderen Fachgesellschaft inklusiv der Einbindung von Referenten geplant. Die vereinbarte Kooperation schließt zudem eine Zusammenarbeit insbesondere in den Bereichen der digitalen Bildverarbeitung, Big data, Al, Texturanalytik und -extraktion sowie der Schaffung gemeinsamer IT-Platt-

formen mit ein.

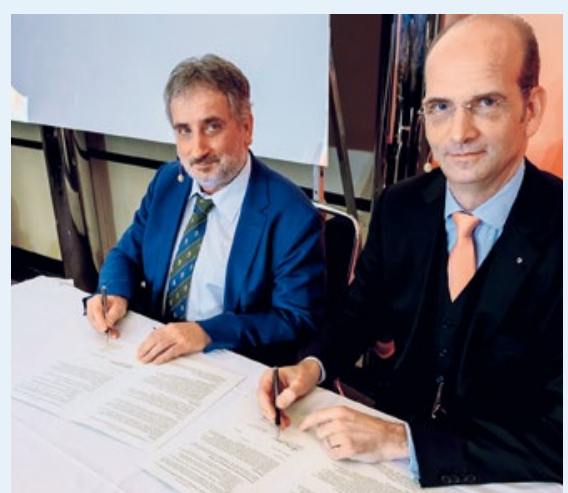

Seitens der DGN wurde die Vereinbarung von unserem Präsidenten Prof. Dr. Bernd Joachim Krause (Rostock) und seitens der DGP von Prof. Dr. Kurt Werner Schmid (Essen) unterzeichnet (Quelle: Frank Pusch, Bremen). 